\title{
Where Is the Clean Air? A Bayesian Decision Framework for Personalised Cyclist Route Selection Using R-INLA*
}

\author{
Laura C. Dawkins ${ }^{\dagger}$, Daniel B. Williamson ${ }^{\dagger, \ddagger}$, Kerrie L. Mengersen ${ }^{\S}$, Lidia Morawska \\ Rohan Jayaratne ${ }^{\mathbb{I}}$, and Gavin Shaddick ${ }^{\dagger, \ddagger}$
}

\begin{abstract}
Exposure to air pollution in the form of fine particulate matter $\left(\mathrm{PM}_{2.5}\right)$ is known to cause diseases and cancers. Consequently, the public are increasingly seeking health warnings associated with levels of $\mathrm{PM}_{2.5}$ using mobile phone applications and websites. Often, these existing platforms provide one-size-fits-all guidance, not incorporating user specific personal preferences.
\end{abstract}

This study demonstrates an innovative approach using Bayesian methods to support personalised decision making for air quality. We present a novel hierarchical spatio-temporal model for city air quality that includes buildings as barriers and captures covariate information. Detailed high resolution $\mathrm{PM}_{2.5}$ data from a single mobile air quality sensor is used to train the model, which is fit using R-INLA to facilitate computation at operational timescales. A method for eliciting multi-attribute utility for individual journeys within a city is then given, providing the user with Bayes-optimal journey decision support. As a proof-of-concept, the methodology is demonstrated using a set of journeys and air quality data collected in Brisbane city centre, Australia.

\section{Introduction}

Air pollution is a major environmental risk to health, estimated to cause 4.2 million premature deaths worldwide annually (World Health Organization, Accessed: 27-11-2018), and 3000 deaths each year in Australia (Australian Institute of Health and Welfare, 2016). Exposure to fine particulate matter (2.5 microns or less in diameter, abbreviated to $\mathrm{PM}_{2.5}$ ) is known to causes cardiovascular and respiratory diseases, and cancers (Sava and Carlsten 2012; Hoek et al. 2013; Loomis et al. 2013).

The general public is becoming increasingly aware of the negative health impacts associated with exposure to $\mathrm{PM}_{2.5}$. As a consequence there is an increasing demand for air quality information and warnings via online web-based tools and mobile phone

\footnotetext{
*This article was first posted without an Acknowledgements section. The Acknowledgements section was added on 8 January 2020.

${ }^{\dagger}$ College of Engineering, Mathematics and Physical Sciences, University of Exeter, UK, lauradawkins@hotmail.co.uk

¥The Alan Turing Institute, British Library, London, UK

$\S$ Science and Engineering Faculty, Queensland University of Technology, Australia

IInternational Laboratory for Air Quality and Health, Queensland University of Technology, Australia
} 


\section{A Bayesian Decision Framework for Personalised Cycle Route Selection}

applications. In most cases, these websites and applications provide generic, one-sizefits-all air quality measurements or warnings for a given location, either based on a single nearby air quality monitoring station, a numerical weather prediction model, or a network of stationary monitoring stations used in combination with a dispersion model, land use regression model and/or satellite data. Examples of such platforms include the Queensland Government live hourly air quality data table (Queensland Government, Accessed: 14/12/2018), Plume (Plume Labs, Accessed: 27-11-2018), BreezoMeter (BreezoMeter, Accessed: 27-11-2018) and MappAir (MappAir, Accessed: 27-11-2018). In many cases these air quality warning platforms provide associated health warnings, often based on the national Air Quality Index (AQI) guidelines which indicates, for example, when it is best to avoid certain activities outside. Again, however, these health warnings are not specific to the user and no platforms consider other parameters of importance, for example the inconvenience of avoiding highly polluted areas.

Here we present a novel approach for combining Bayesian decision theory and hierarchical Bayesian spatio-temporal modelling to inform a personalised air quality warning tool, able to provide Bayes-Optimal decision support about which route a given user should take through a city.

In order to develop a personalised decision making framework, a multiattribute utility function must capture a user's preferences over competing rewards (Smith, 2010). For example, suppose two potential journeys exist from A to B, one characterising a highly polluted, short route and an alternative characterising a cleaner, longer route. Suppose a given individual is most concerned with arriving at their destination as quickly as possible while another considers the impact on their health to be most important: the optimal route for each individual is likely to be different. Here we present a method for eliciting these complex preferences about competing journeys, and show how they can be used to inform a Bayes-Optimal route for a given individual. Specifically, preferences about three decision-relevant journey attributes are considered: a measure of the health impact of exposure to $\mathrm{PM}_{2.5}$ along the journey, the journey time in minutes, and a measure of the journey enjoyment. In a similar way, Economou et al. (2016) used a Bayesian decision analysis approach within an environmental hazard application to provide personalised meteorological natural hazard warnings for individual stakeholders. Predominantly, however, such theory has not been utilised in this field. A detailed description of Bayesian decision analysis can be found in Smith (2010). Here, as a proofof-concept, we focus on developing a Bayesian decision theoretic tool for personalised cycle route selection in Brisbane city centre.

In addition to capturing users preferences, we require a probability model for $\mathrm{PM}_{2.5}$ exposure for any route that a user may choose. To achieve this, we develop a Bayesian hierarchical spatio-temporal model for $\mathrm{PM}_{2.5}$, able to accurately predict $\mathrm{PM}_{2.5}$ exposure along potential routes within the geographical domain. Stationary air quality monitoring stations are limited in their spatial coverage and are therefore unable to represent the spatial variability relevant for assessing personal exposure to pollution along a route. Consequently, in recent years mobile air quality monitors have become increasingly used as an additional tool to acquire air quality data at high spatial and temporal resolutions, and at locations most relevant for personal exposure (Van den Bossche et al., 2015). This 
increase in mobile air quality sensing comes after recent improvements in low cost mobile monitors. These include the Airoflex monitor, used by Elen et al. (2013) to monitor ultra fine particles (UFP) and black carbon (BC) in Antwerp, Belgium, and the KOALA (Knowing Our Ambient Local Air-quality) monitor, recently developed by the International Laboratory of Air Quality and Health (ILAQH), Australia, used to monitor air quality at the 2018 Commonwealth Games (ILAQH, Accessed: 28-11-2018). A comprehensive overview of the rapidly growing body of literature on mobile air quality sensing can be found in Van den Bossche et al. (2015), in combination with Xie et al. (2017).

Hierarchical Bayesian models provide a useful framework for representing complex air quality data. A number of examples of such models exist for stationary air quality monitoring stations, for example Cocchi et al. (2007), Sahu and Bakar (2012) and Li et al. (2013). However more recently, in response to the growing recognition of mobile air quality monitors, Del Sarto et al. (2016) developed a hierarchical Bayesian spatiotemporal modelling approach for representing such data. This model uses a three level hierarchy in which the mobile observations are modelled in the first stage of the hierarchy, and a latent spatio-temporal process, defined on a discrete space-time grid, is considered in the following stages, also capturing covariate information. As such, this form of hierarchical Bayesian model is able to give a detailed representation of air quality at the most relevant locations (i.e. where people actually travel), whilst also providing a comprehensive quantification of uncertainty. This allows for more specific and accurate personal guidance on where and when to go to minimise exposure to harmful air pollution, compared to current air quality warning platforms. In addition, the recent advancements in computational tools for implementing the Integrated Nested Laplace Approximation (INLA) approach for Bayesian inference (Rue et al., 2009), a computationally efficient alternative to Markov Chain Monte Carlo (MCMC) methods, facilitates the application of such complex hierarchical Bayesian models at operational timescales, for example to provide real-time air quality warnings. In addition, rather than assuming a stationary spatial field our INLA air quality model incorporates an adaptation of the barrier model of Bakka et al. (2018), originally developed to model coastlines, to better represent the way in which high-rise buildings block the flow of air within the city, causing non-stationarity.

Following this we explore approaches for equating exposure to $\mathrm{PM}_{2.5}$ along a given route to the associated impact on health, the first decision-relevant journey attribute. The other two decision-relevant journey attributes, journey time and enjoyment, are estimated using Google maps and Google route planner. We then present an approach for eliciting the personal preferences of the user, including an R Shiny web interface.

We present a proof-of-concept cycle route selection tool tailored to comparing two alternative routes through Brisbane city centre, shown in Figure 1, at two different times of the day. These routes have differing characteristics to demonstrate how distinct personal preferences will result in a particular cycle route selection. Route 1 (purple) is predominantly cycled next to the river along a scenic cycle path, reducing exposure to $\mathrm{PM}_{2.5}$, increasing journey enjoyment, but also increasing journey time, while route 2 (blue) is shorter and quicker but predominantly cycled on the busy roads within the Central Business District (CBD), increasing exposure to $\mathrm{PM}_{2.5}$ and reducing journey enjoyment. 
64 A Bayesian Decision Framework for Personalised Cycle Route Selection

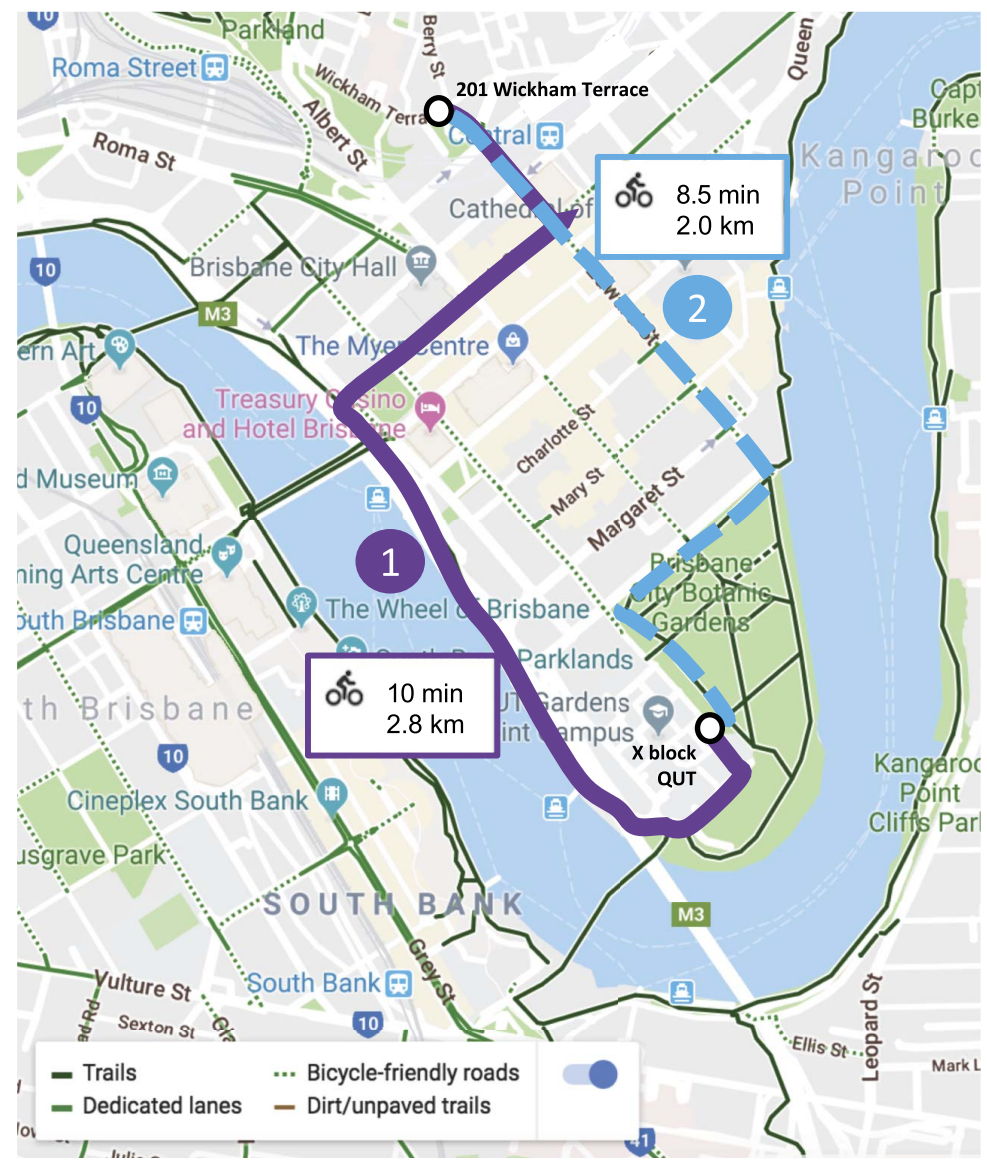

Figure 1: A google map showing the two routes used within this proof-of-concept study to demonstrate how our method optimises different journeys based on differing personal preferences. These two routes go between X block at Queensland University of Technology (QUT) and Wickham Terrace to the north of the Central Business District (CBD) taking different cycle friendly paths.

The remaining paper is organised as follows. In Section 2 we present our approach to quantifying the decision-relevant attributes for our proof-of-concept application in Brisbane, including a description of the mobile monitor data collection scheme in Section 2.1, the development of the Bayesian spatio-temporal model for mobile air quality monitor data using INLA in Section 2.2, and the subsequent representation of the three decision-relevant journey attributes, in particular how $\mathrm{PM}_{2.5}$ exposure is related to health in Section 2.3. Section 3 outlines the method for eliciting user-specific preferences for the Bayesian decision framework, followed by the case study demonstration of the decision framework in Section 4. Finally Section 5 concludes. 


\section{Quantifying Decision-Relevant Journey Attributes}

Let $d$ represent the decision to choose a particular cycle route; $\theta$ represent the exposure to $\mathrm{PM}_{2.5}$ along this chosen route; $y$ denote all available relevant data; and $r(d, \theta)=$ $\left(r_{1}(d, \theta), r_{2}(d, \theta), r_{3}(d, \theta)\right)$ represent the three decision-relevant journey attributes for the chosen route: health impact of exposure to $\mathrm{PM}_{2.5}$, journey time, and journey enjoyment respectively. Bayesian decision theory shows that the optimal decision $d^{*}(y)$ is the one that maximises the expected utility, which, if mutual independence between attributes is assumed, can be written as:

$$
\bar{U}(d)=\int_{\theta}\left(\sum_{i=1}^{3} k_{i} U_{i}\left(r_{i}(d, \theta)\right)\right) p(\theta \mid y) d \theta,
$$

where $U_{i}$ is a utility function, and $k_{i}$ the criterion weight for decision-relevant journey attribute $i$. The criterion weight, $k_{i}$, characterises how important the decision maker considers attribute $i$ to be in relation to the other attributes, such that $\sum_{i} k_{i}=1$, while the utility function, $U_{i}$, represents how risk adverse the decision maker is to incurring a bad outcome of attribute $i$ (see Smith (2010) and references there in for more detail). The assumption of mutual independence is a made here to allow the paper to focus on demonstrating the framework, however an alternative dependence structure could be modelled using Bayesian decision theory. This is discussed further in Section 5.

In Section 3 we develop a method for eliciting $U_{i}$ and $k_{i}$ for $i=1,2,3$ from the user. Firstly, however, in this section we develop our probability model for $\theta\left(\mathrm{PM}_{2.5}\right.$ exposure), including an efficient method for inference given mobile air quality monitor data, $y$. Following this, an approach for relating exposure to $\mathrm{PM}_{2.5}$ to adverse health impacts is developed, quantifying $r_{1}$. The other decision-relevant attributes, journey time and journey enjoyment $\left(r_{2}\right.$ and $\left.r_{3}\right)$, are obtained without requiring the development of a statistical model. Journey time is manually extracted from Google Maps, while journey enjoyment is calculated as the proportion of the journey time spent cycling off-road (i.e. not on a section of the route within the CBD).

\subsection{Data}

The air quality data used within this application were collected using a compact, stateof-the-art, portable air quality monitor $(26 \times 21 \times 11 \mathrm{~cm}$ ), KOALA (Knowing Our Ambient Local Air-quality), developed by scientists at the International Laboratory of Air Quality and Health (ILAQH) at Queensland University of Technology (QUT), in Brisbane, Australia, as part of a larger project on establishing advanced networks of air quality sensing and analysis (ILAQH, Accessed: 28-11-2018). The KOALA monitor includes two low-cost sensors that measure $\mathrm{PM}_{2.5}$ and carbon monoxide concentrations in real time. Readings were obtained at 5 -second intervals and stored on a built-in SD card for later download and analysis.

For this study the monitor was attached to the front basket of a bicycle and used measure $\mathrm{PM}_{2.5}$ concentration along roads and cycle paths within Brisbane city centre. A GPS tracker was attached to the bike alongside the KOALA monitor, recording its 
location at approximately equivalent time intervals. In this way the mobile monitor was able to provide data at a high spatial resolution, in locations relevant for presenting personal exposure to $\mathrm{PM}_{2.5}$ while cycling through the city centre.

The high temporal variability of urban air quality means that achieving a representative data sample requires careful consideration of three factors: the geographical location of the data collection route, the most relevant times at which to collect the data (i.e. which days of the week and times of the day?), and the frequency of repeated data collection laps.

Primarily, since this study is focused on developing a tool for cyclists and the data was to be collected on-bike, the data collection route was restricted to "cycle friendly" routes only, as defined by Google Maps. In addition, the physical exertion involved in collecting the data restricted the route to an approximately $2 \mathrm{~km}^{2}$ central region of Brisbane city (longitude range 153.0193:153.0316, and latitude range -27.48214:-27.46464). As in previous similar studies using mobile air quality monitors such as Van den Bossche et al. (2015) and Peters et al. (2013), the route was designed to encompass the varying terrain and road types within the city centre. Specifically here, this meant ensuring the route included the on-road bike paths within the Central Business District (CBD); the pedestrianised routes through the Botanic Gardens, situated to the south of the CBD; the Bicentennial bike-way running along the Brisbane river to the west of the CBD; and the pedestrianised route on the other side of the river, known as South Bank. In addition, the route was devised to encompass the most popular cycle routes within these four regions, based on the freely available STRAVA (social fitness network) heat map, a visualisation of the frequency with which roads are used by their cyclist subscribers (Strava, Accessed: 14-01-2019). Finally, the route was designed to include likely destinations for cyclists journeying into the city centre, namely bicycle parking racks and the bike stations of the local bike sharing scheme, CityCycle. The final route, as shown in Figure 2, was approximately $8 \mathrm{~km}$ in length, taking roughly 35 minutes to complete.

The temporal data collection schedule was designed to capture the days of the week and times of the day most frequented by cyclist. Mateo-Babiano et al. (2016) presented an analysis of the CityCycle usage habits of people living and working in Brisbane between May 2012 and April 2013. Mateo-Babiano et al. (2016) identified a general peak in CityCycle usage on weekdays during the morning commute 6:00-10:00, and the evening commute 16:00-19:00, and on weekends during the middle of the day 10:0016:00. Similarly, as an ongoing initiative to improve cycling infrastructure in Brisbane the city council have been carrying out cyclist counts throughout the city, published by the Brisbane Times, identifying the same peaks in cyclist numbers (Brisbane Times, Accessed: 14-01-2019). They provide specific counts for Brisbane CBD (separate from other regions of the city), indicating a slightly later peak in the morning commute (6:3010:30) and a slightly earlier peak in the evening commute (15:30-18:30), capturing how the $\mathrm{CBD}$ is the most common place of work and is therefore the end point of morning commute and start point of the evening commute. In addition, this count revealed that Tuesdays and Thursdays have slightly higher cyclist numbers than other weekdays, and Sundays are marginally more popular with cyclists than Saturday. 
(a)

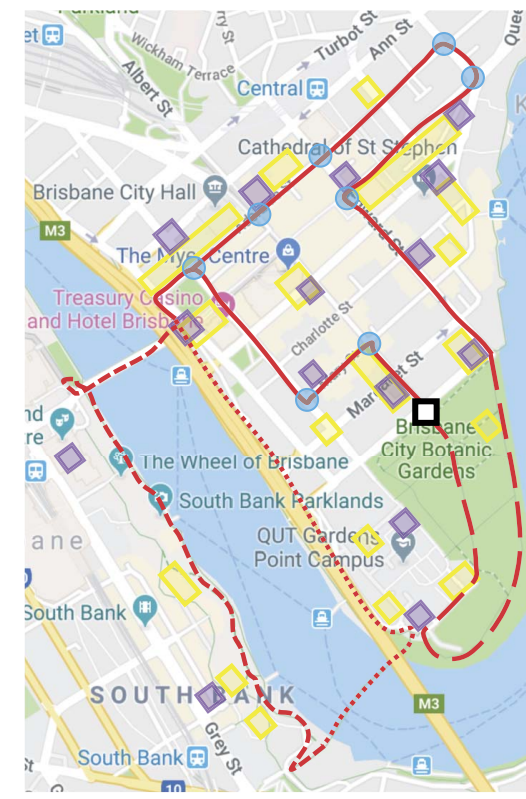

(b)

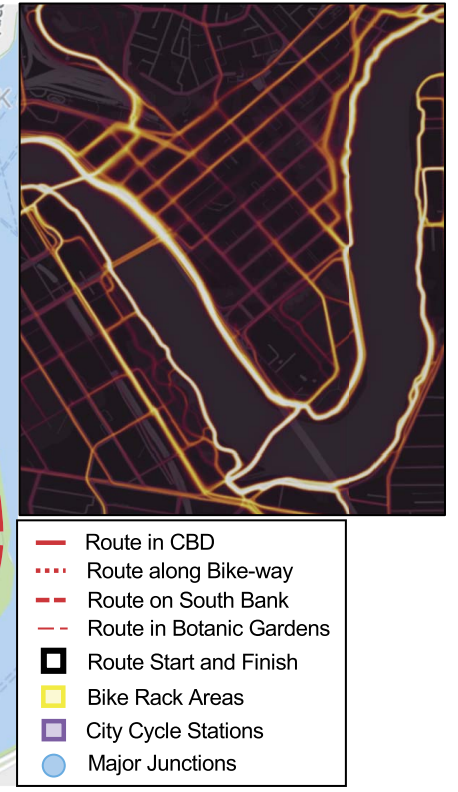

Figure 2: (a) The cycle route devised for data collection, indicating the different regions of Brisbane city centre (CBD, bike-way, South Bank and Botanic Gardens) and the location of bike racks and CityCycle docking stations, (b) The STRAVA heat map for Brisbane city centre.

The data collection schedule on a given day was restricted by the battery life and charging time needed for the KOALA monitor to operate. Once fully charged the monitor could run for 3 hours at a time and then required another 4 hours to charge in between runs. Following this restriction and the peak cycling times identified by MateoBabiano et al. (2016) and Brisbane City Council, the data collection on weekdays was organised into 4 time slots: one early morning commute (7:00-8:30), one later morning commute (8:30-10:00), one early evening commute (15:30-17:00) and one later evening commute (17:00-18:30). Data collection on the weekend was organised into 2 time slots: early leisure time (10:00-11:30) and later leisure time (11:30-13:00).

Finally, the required number of repeated data collection laps was considered. As identified by Van den Bossche et al. (2015) and Peters et al. (2013), high resolution spatial mapping of air quality requires repeated mobile monitor measurements. Peters et al. (2013) concluded that a limited set of mobile measurements (20-24, and for some streets as few as 10) made it possible to map air quality, while Van den Bossche et al. (2015) identified that, depending on the location, 24-94 (median 41) repeated measurements were required. Here, data collection was carried out by a team of 16 volunteers, restricting the availability of participation, within a limited project time frame. Following the Brisbane cycle count results, data collection was allocated to Tuesday, Thursday and 


\section{A Bayesian Decision Framework for Personalised Cycle Route Selection}

Sunday during a short period of 2 weeks running from Thursday $10^{\text {th }}$ May-Thursday $24^{\text {th }}$ May 2018, encompassing 2 Tuesdays, 3 Thursdays and 2 Sundays, hence 7 days in total. To increase the number of repeated laps and hence the representativeness of the sample, the route was completed twice within each of the time slots on each of the data collection days. This resulted in a total of 48 laps of the data collection route, within 24 time slots, over 7 days within a 2 week period in May 2018, made up of a total of 15847 data points. These 48 laps are shown in Figure 3. Following data collection, to ensure $\mathrm{PM}_{2.5}$ was recorded along cycle routes, ArcGIS was used to move GPS locations associated with each data point to the nearest location along the route.

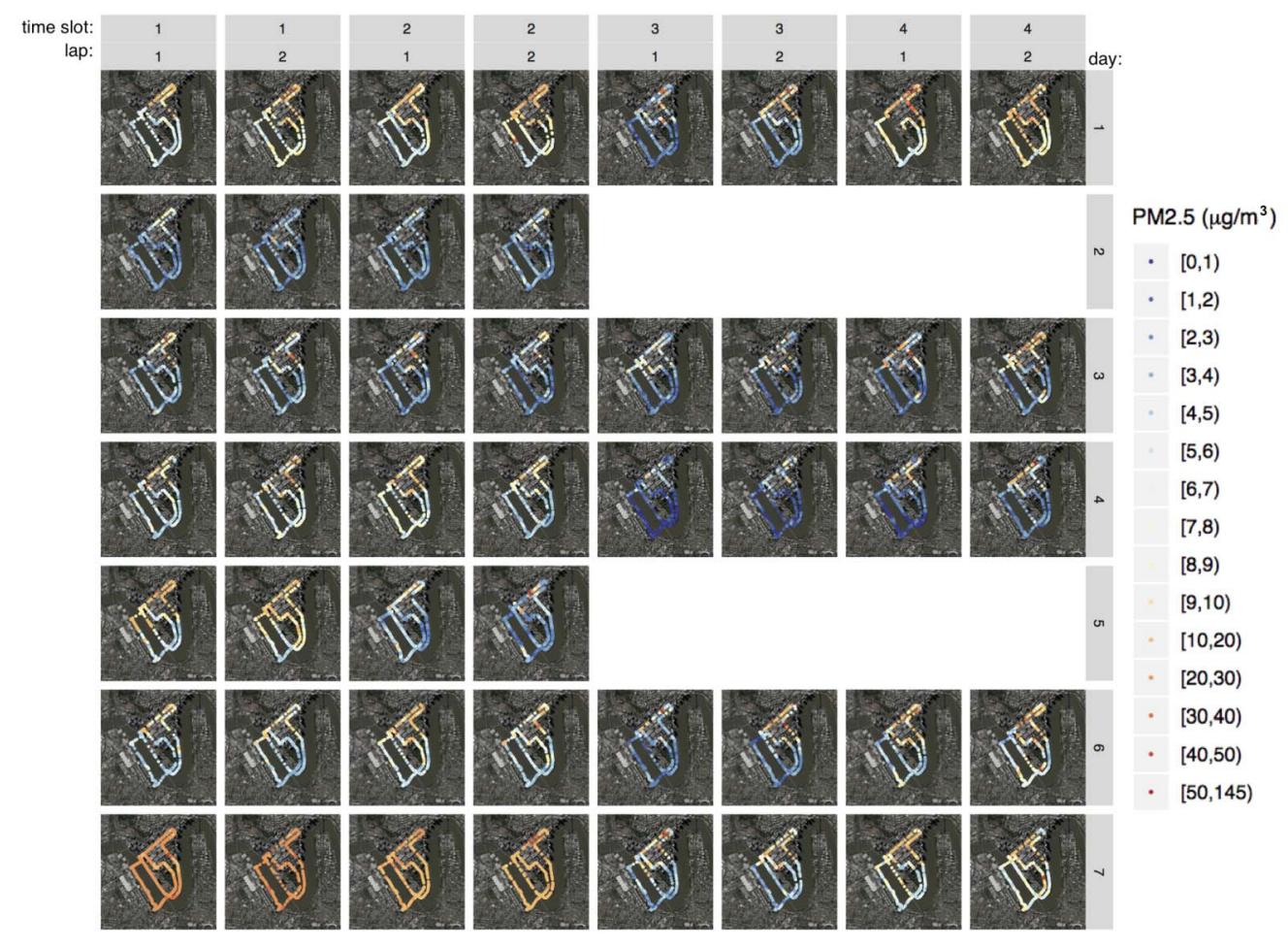

Figure 3: The $48 \mathrm{PM}_{2.5}$ laps of data collected using the KOALA mobile air quality monitor. These 48 laps were collected over 24 time slots, (1-4) on weekdays and (1-2) on weekends [columns], and 7 days in May 2018 (Thursday 10/05/2018, Sunday $13 / 05 / 2018$, Tuesday $15 / 05 / 2018$, Thursday $17 / 05 / 2018$, Sunday $20 / 05 / 2018$, Tuesday 22/05/2018, Thursday 24/05/2018) [rows].

Further data used within this study consisted of 5 minute average $\mathrm{PM}_{2.5}$ concentrations and hourly average meteorological measurements (temperature, wind direction, wind speed and humidity) from three stationary monitoring stations located in Wooloongabba, South Brisbane and the Queensland University of Technology campus, available from the Queensland Government (Queensland Government, 
Accessed: 14/12/2018). In addition, electronic 30 minute total traffic counts at four intersections within the city centre (Adelaide Street - Wharf Street, Elizabeth Street George Street, Elizabeth Street - Edward Street and Mary Street - Albert Street) were kindly provided by Brisbane City Council. For each variable described above the available data was used to calculate an average for each of the 1.5 hour time slots within the data collection schedule. Finally, geographical variables akin to those used in air quality land use regression models (e.g. Hoek et al., 2008) were derived using ArcMap, including, for each data point, the distance from a major junction and distance from the river. The junctions considered 'major' were those at which the cyclists were often made to wait at traffic lights for a long period of time, shown in Figure 2.

\subsection{A Spatio-temporal Model for Mobile Air Quality Monitor Data Using the INLA Barrier Model}

We develop a spatial-temporal model for mobile air quality data, building upon the model presented by Del Sarto et al. (2016). We consider a model structure in which realisations of the spatial-temporal process are indexed by a nested time structure and spatial grid. That is, for day $d=1, \ldots, D$, time slot $t=1, \ldots, T_{d}$ and spatial grid cell $s=1, \ldots, S, Z_{d t s}$ represents the spatial process at the spatio-temporal point $d t s$. As the mobile data collection route is completed, multiple observations are made within the vicinity, and hence are representative of each of these spatio-temporal points. Therefore, let $Y_{d t s j}$ for $j=1, \ldots, J_{d t s}$ represent these $J_{d t s}$ repeated observations at the spatiotemporal point $d t s$. The mobile data collection routes vary slightly each time they are completed, hence $J_{d t s}$ can be different for each grid point $d t s$. Similar to Del Sarto et al. (2016), we model this data using a Hierarchical Bayesian framework, in which each nested index is modelled as a different layer of the hierarchy. This allows us to incorporate covariates at each level of the model and to model different sources of error separately. Let the mobile air quality monitor observations be denoted $Y_{i}$, for $i=1, \ldots, d t s j, \ldots, N$ (where $\left.N=D T_{D} S J_{D T_{D} S}\right)$, then,

$$
\begin{aligned}
Y_{i} & =\mu_{i}+e_{i}, \\
\mu_{i}=\mu_{d t s j} & =Z_{d t s}+\beta_{0}+\beta X_{d t s j}+\xi_{d t s j}, \\
Z_{d t s} & =Z_{d t}+\tilde{\beta} \tilde{X}_{d t s}+\epsilon_{d t s}, \\
Z_{d t} & =Z_{d}+\phi_{d t}, \\
Z_{d} & =Z+\beta^{\prime} X_{d}^{\prime}+\psi_{d}, \\
Z & \sim G F(0, \Sigma),
\end{aligned}
$$

where:

- $Y_{i}$ is the $i^{\text {th }}$ observation, $i=1, \ldots, d t s j, \ldots, N$,

- $\mu_{i}$ is the mean, and $e_{i}$ the error, associated with observation $i$,

- $Z_{d t s}$ is the latent process at spatio-temporal grid point $d t s$, 


\section{A Bayesian Decision Framework for Personalised Cycle Route Selection}

- $\beta_{0}$ is the overall intercept coefficient, constant for all observations,

- $X_{d t s j}$ is a $p \times 1$ vector of covariates available for each observation,

- $\beta$ is the $p \times 1$ vector of coefficients associated with these $p$ covariates,

- $\xi_{d t s j}$ is the error within spatial locations, for example in our data accounting for the difference in the two laps of the data collection route within each time slot,

- $Z_{d t}$ is the latent process at time $d t$,

- $\tilde{X}_{d t s}$ is a $\tilde{p} \times 1$ vector of covariates available on the same resolution as the spatiotemporal points $d t s$ only,

- $\tilde{\beta}$ is the $\tilde{p} \times 1$ vector of coefficients associated with these $\tilde{p}$ covariates,

- $\epsilon_{d t s}$ is the error among spatial locations,

- $Z_{d}$ is the latent process on day $d$,

- $\phi_{d t}$ is the error among time slots,

- $Z$ is a zero mean non-stationary Gaussian random field with spatial covariance matrix $\Sigma$, defined over the spatial field $s=1, \ldots, S$,

- $X_{d}^{\prime}$ is a $p^{\prime} \times 1$ vector of covariates available for each day only,

- $\beta^{\prime}$ is the $p^{\prime} \times 1$ vector of coefficients associated with these $p^{\prime}$ covariates,

- $\psi_{d}$ is the error among days.

This model differs from that of Del Sarto et al. (2016) in a number of ways. An additional level is added to the top of the hierarchy such that each observation has an associated error term. This additional error term is necessitated by the repeated lap structure of the data collection route, which results in data points recorded approximately an hour apart being considered to be at the same spatio-temporal grid point. In addition, the auto-regressive (AR) temporal component of the Del Sarto et al. (2016) model is omitted from this model structure. Again, this is due to the differing temporal structure of our data. Del Sarto et al. (2016) consider hourly data throughout a number of consecutive days, while here, we consider two/four disjointed 1.5 hour time slots during non-consecutive weekends/weekdays. Further, we include an additional level at the bottom of the hierarchy to capture the variability between days, in terms of covariates available for each day only and the error among days.

Moreover, rather than using MCMC as in Del Sarto et al. (2016), here the Gaussian random field (GF) $Z(s)$ is modelled using a computationally efficient alternative to MCMC, the Integrated Nested Laplace Approximation (INLA), Stochastic Partial Differential Equation (SPDE) approach of Lindgren and Rue (2011). The advantage of this approach, compared to using an MCMC approach, is its applicability to very high dimensional data and reduced computational time and expense. This facilitates Bayesian 
model fitting in real time, as would be required for making air quality predictions within a web or mobile phone application.

Finally, unlike the model of Del Sarto et al. (2016), the non-stationarity of the spatial domain resulting from topographical variability of multi-storey high-rise buildings, is captured by using the non-stationary barrier model extension of the INLA framework, originally developed by Bakka et al. (2019) to model coast lines. A physical barrier is specified, and the solutions to the SPDEs representing the barriered and non-barriered regions, as specified in Bakka et al. (2019), are obtained. As in the stationary INLA model, this is achieved by approximating the SPDE solution using a finite element method, whose elements are vertices of Delauney triangulations over the spatial domain (Shaddick and Zidek, 2014). A full description of the INLA SPDE approach can be found in Lindgren and Rue (2011), and a useful summary is given by Shaddick and Zidek (2014). The barrier model is specified in full in Bakka et al. (2018) and can be implemented using the R-INLA R package, instructions and examples of which are available within comprehensive tutorials at http://www.R-INLA.org/.

\section{Modelling $\mathrm{PM}_{2.5}$ in Brisbane City Centre}

Here, we model $\mathrm{PM}_{2.5}$ in Brisbane city centre, over the data collection schedule discussed in Section 2.1. Similar to many examples in the literature including Del Sarto et al. (2016), we model the $\log$ transformation of $\mathrm{PM}_{2.5}$, which has been shown to be well represented by the Normal distribution. In addition, we apply this log transform to $\mathrm{PM}_{2.5}+1$ to ensure non-infinite transformed values.

Based on our previous Brisbane air pollution work (e.g. Morawska et al. 2002), other relevant literature, and available relevant data (described in Section 2.1), we conducted a preliminary exploratory data analysis to identify relevant covariate information to include within the different levels of our Bayesian Hierarchical model (2). This exploration involved both the study of scatter plots of possible relevant relationships within the data, and model comparison using the Watanabe-Akaike Information Criterion (WAIC), as recommended by Gelman et al. (2014) and included within the R-INLA package, removing and including each covariate to explore all relevant combinations. Future work could involve incorporating model selection within the model fitting using, for example, shrinkage priors on the regression coefficient parameters. This is, however, beyond the scope of this study.

Exploration of covariates associated with each observation, $X_{d t s j}(2 \mathrm{~b})$, included geographical measures: distance to the nearest major junction (see Figure 2) and distance to the river, obtained using ArcMap, and 30 minute resolution traffic counts at 4 major intersections. The interaction between traffic counts and a factor representing the time of day (i.e. which time slot) was included in the exploration to characterise how traffic flows in and out of the city along different roads.

Relevant covariates available on the resolution of the imposed spatio-temporal grid, $X_{d t s}$ (2c) included the background level of $\mathrm{PM}_{2.5}$, represented by the level at the nearest stationary monitoring station located in South Brisbane, and meteorological conditions within the time slot. In a previous study of Brisbane air quality, Morawska et al. (2002) 


\section{A Bayesian Decision Framework for Personalised Cycle Route Selection}

identified a notable diurnal cycle in wind direction in Brisbane city centre, owing to its geographical location (coastline close by to the east). In the morning the winds generally come from the west, blowing polluted air from the motorway onto the CBD, while in the afternoons, the sea breeze from the east blows this polluted air away from the CBD. Wind direction as a factor characterising wind from the east/west was therefore included in the exploratory data analysis. In addition, the influence of boundary layer height, often determined from temperature, humidity and wind speed, on air pollution concentration is well documented (Zhang et al., 2014; Kumar Mehta et al., 2017). During morning rush hour, which is just after sunrise and hence during relatively cool conditions, the vehicle emissions mix into a shallow boundary layer, thus increasing pollution. As the day warms up, the boundary layer height increases so emissions are mixed into a larger volume of air. Thus the effects of evening commuter emissions are not as severe as the effects of morning commuter emissions; this can be seen in Figure 3. To represent this phenomenon, temperature, humidity and wind speed were included in the model exploratory analysis. Based on insights from previous work, it was expected that the effect of meteorological parameters would be less pronounced within the CBD, where roads are shielded by buildings. Following this insight, the interaction between meteorological variables and a location factor (in CBD/not in CBD) were also included in the exploration. Finally, a factor characterising the 'day type', as either weekday or weekend was explored as a covariate available at the day level, $X_{d}^{\prime}(2 \mathrm{e})$.

After conducting the exploratory data analysis, the resulting covariates included in the model are as follows (with hierarchy within the model shown in square brackets):

- Distance from a major junction $(2 \mathrm{~b})$

- Distance from the river $(2 \mathrm{~b})$

- Electronic traffic counts at 4 intersections in Brisbane CBD and the interaction between these and the categorical variable for time of day (time slots 1-4) (2b)

- Average $\mathrm{PM}_{2.5}$ at the South Brisbane monitoring station during the proceeding 1.5 hours $(2 \mathrm{c})$

- Meteorological variables and the interaction between these and the CBD binary variable $(0=$ not in $\mathrm{CBD}, 1=$ in $\mathrm{CBD})(2 \mathrm{c})$

- Temperature at the Brisbane CBD monitoring station

- Wind Direction at the Brisbane CBD monitoring station, as a binary variable $(0=$ East, $1=$ West $)$

- Wind speed at the South Brisbane monitoring station

- Humidity at the South Brisbane monitoring station

- Day type, as a binary variable $(0=$ weekday, $1=$ weekend $)(2 \mathrm{e})$

The regression coefficients $\left(\beta_{0}, \beta, \tilde{\beta}, \beta^{\prime}\right)$ are each assigned a Normal prior (Gelman et al., 2008). Where the exploratory data analysis indicated a clear negative or positive 
relationship between $\log \left(\mathrm{PM}_{2.5}+1\right)$ and the covariate, the corresponding prior mean for that regression coefficient is set to -1 or 1 respectively, such that:

$$
\begin{aligned}
& \beta_{0} \sim N\left(0,0.001^{-1}\right), \\
& \beta_{k} \sim N\left(c_{k}, 0.001^{-1}\right) \quad \text { for } \mathrm{k}=1, \ldots, \mathrm{p}, \\
& \tilde{\beta}_{\tilde{k}} \sim N\left(\tilde{c}_{\tilde{k}}, 0.001^{-1}\right) \quad \text { for } \tilde{k}=1, \ldots, \tilde{p}, \\
& \beta_{k^{\prime}}^{\prime} \sim N\left(c_{k^{\prime}}^{\prime}, 0.001^{-1}\right) \text { for } k^{\prime}=1, \ldots, p^{\prime} \text {, }
\end{aligned}
$$

where $c_{k}=(-1,1,1,1,1,1)$, for distance to major junction, distance to river and all four traffic counts respectively, $\tilde{c}_{\tilde{k}}=(1,-1,1,-1,1)$, for $\mathrm{PM}_{2.5}$ at South Brisbane in the preceding 1.5 hours, temperature, wind direction from the west, wind speed and humidity respectively, and $c_{k^{\prime}}^{\prime}=1$, associated with the weekday day type factor. All other prior means are set at zero. In all cases, the prior precision (inverse variance) is set to 0.001 (the INLA default). Alternative precision parameters (0.01 and 0.1 ) were explored for sensitivity, resulting in indistinguishable posterior distributions for the model parameters (shown in the table given in the Supplementary Material (Dawkins et al., 2020)).

A zero mean normal prior is assigned to each of the error terms in the model. The precision of each of these priors is assigned a log-gamma hyper prior with shape and rate parameters $a$ and $b$, respectively.

$$
\begin{array}{rlrl}
e_{i} & \sim N\left(0, \sigma_{e}^{2}\right) & & 1 / \sigma_{e} \sim \log \text {-gamma }(a, b), \\
\xi_{d t s j} \sim N\left(0, \sigma_{\xi}^{2}\right) & & 1 / \sigma_{\xi} \sim \log \text {-gamma }(a, b), \\
\epsilon_{d t s} & \sim N\left(0, \sigma_{\epsilon}^{2}\right) & 1 / \sigma_{\epsilon} \sim \log \text {-gamma }(a, b), \\
\phi_{d t} \sim N\left(0, \sigma_{\phi}^{2}\right) & 1 / \sigma_{\phi} \sim \log \text {-gamma }(a, b), \\
\psi_{d} \sim N\left(0, \sigma_{\psi}^{2}\right) & 1 / \sigma_{\psi} \sim \log \text {-gamma }(a, b) .
\end{array}
$$

As described in the R-INLA multilevel model tutorial (Faraway, Accessed: 13/12/2018), the variance of each of these random effects is expected to be lower than the variance of the residuals of the fixed-effects only model. Based on the exploratory data analysis this variance is expected to be approximately equal to 0.2 . These hyper prior parameters are therefore chosen such that the mean of the log-gamma distribution of the precision $\left(\frac{a}{b}\right) 0.2$, hence $a=0.5$ and $b=0.1$. Alternative combinations of these parameters were explored (e.g. $a=1, b=0.2$ ), again making a negligible difference to the resulting model inference.

\section{Model Fitting}

Fitting the INLA barrier model using R-INLA first requires the creation of a mesh of Delauney triangulations, including the specification of a maximum triangle edge length, a model domain boundary and the location of barriers within the domain. Within this study the maximum triangle edge length was specified as $0.2 \mathrm{~km}$ within the inner domain and $0.4 \mathrm{~km}$ in the outer domain, the boundary of the domain was constructed 


\section{A Bayesian Decision Framework for Personalised Cycle Route Selection}

as a polygon encompassing Brisbane city centre, and the barriers were specified as the location of high-rise buildings within the domain. The resulting mesh created using R-INLA is presented in Figure 4, consisting of 1090 triangle vertices.

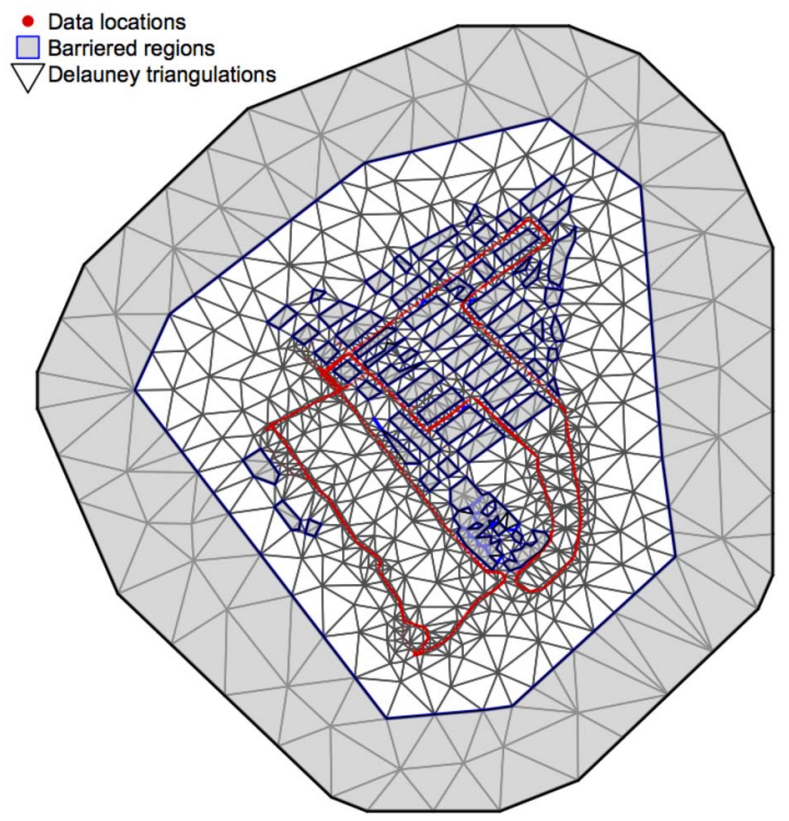

Figure 4: Mesh of Delauney triangulations created using R-INLA to represent Brisbane city centre within the spatial INLA barrier model for $\mathrm{PM}_{2.5}$ in Brisbane city centre.

Using this mesh in combination with the $\mathrm{PM}_{2.5}$ mobile monitor data, covariate data, the model formula specified in (2) and priors specified above, model fitting takes approximately 15 minutes with R-INLA.

The mean and $90 \%$ credible interval for each of the model parameters is summarised in a table given in the Supplementary Material. The mean and standard deviation of the INLA barrier model Gaussian random field (GF), $\mathrm{Z}$ in (2f), is presented in Figure 5. The mean of the GF (Figure 5a) clearly shows how the barriers within the model affect the spatial dependence in the domain, creating the non-stationary structure that would be produced by high-rise buildings. The areas of peak mean pollution are at locations on the busy roads within the $\mathrm{CBD}$ and the barriers create a more physically realistic dependence structure in which high levels of background $\mathrm{PM}_{2.5}$ are funnelled down the urban canyon created by the high-rise buildings. In addition, as would be expected, the lowest levels of background $\mathrm{PM}_{2.5}$ are found in the botanic gardens, and similarly the barriers within the model characterise how the high rise buildings might affect the spatial dependence between the gardens and the nearby roads.

Figure 6 demonstrates how the model represents the observations included within the model fitting. Figure 6 (a) indicates that there is close agreement between true and 

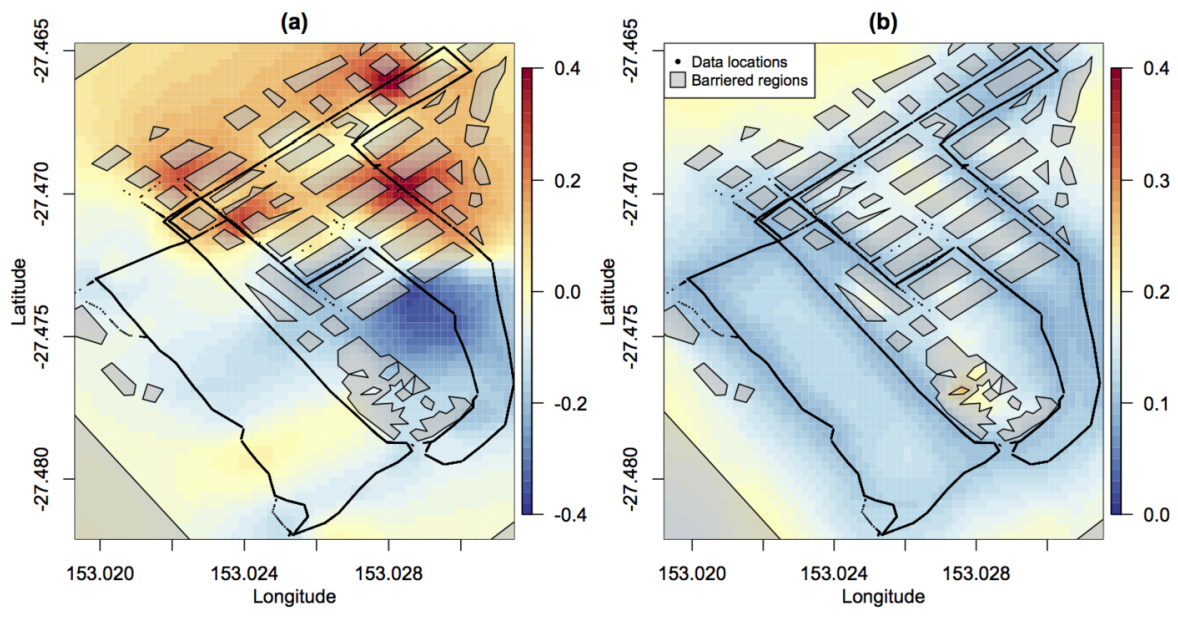

Figure 5: (a) The mean, and (b) standard deviation, of the INLA barrier model Gaussian random field for $\mathrm{PM}_{2.5}$ in Brisbane city centre.

(a)

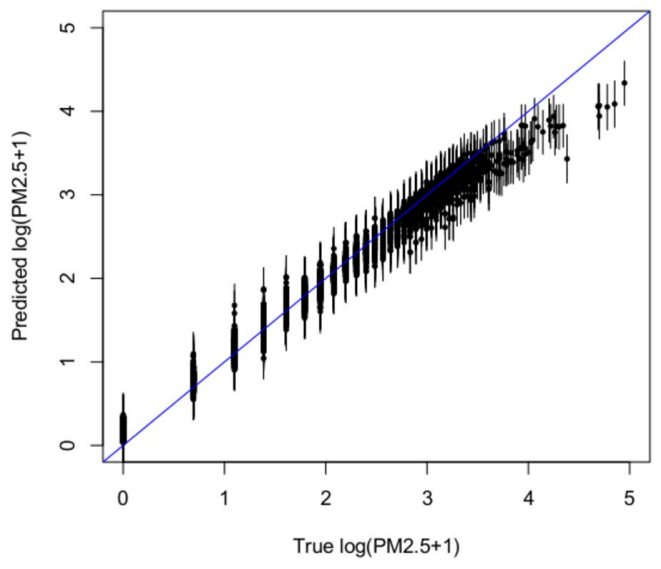

(b)

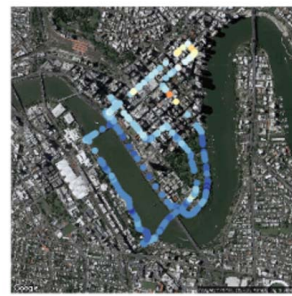

(c)

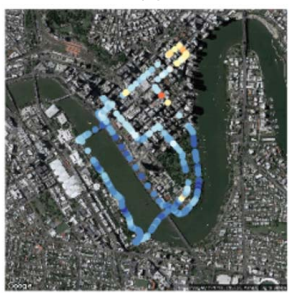

PM2.5 (ug/m3)

- $[0,1)$

- $[1,2)$

- $[2,3)$

- $[3,4)$

- $[4,5)$

- $[5,6)$

$[6,7)$

$77,8)$
$(8,9)$
$(9,10)$

$[9,10)$
$[10,20)$

- $[20,30)$

- $[30,40)$

Figure 6: (a) Scatter plot comparing posterior predictive mean (black points) and $90 \%$ prediction interval (black line) with true $\log \left(\mathrm{PM}_{2.5}+1\right)$ for all mobile air quality monitor observations, and a comparison of (b) the posterior predictive mean and (c) true $\mathrm{PM}_{2.5}$ for one randomly selected lap (Tuesday $15^{\text {th }}$ May 2018, lap 2 in the 8:30-10:00am time slot).

predicted values up to high magnitudes of approximately $\log \left(\mathrm{PM}_{2.5}+1\right)=3.8$, equivalent to $\mathrm{PM}_{2.5}=46 \mu \mathrm{g} / \mathrm{m}^{3}$. The model is less able to represent extreme observed levels of $\mathrm{PM}_{2.5}$. This underestimation is also observable in Figure 6 (b) and (c), where the peak 


\section{A Bayesian Decision Framework for Personalised Cycle Route Selection}

observed $\mathrm{PM}_{2.5}$ in this lap, equal to $34 \mu \mathrm{g} / \mathrm{m}^{3}$, is associated with a posterior predictive mean of $25 \mu \mathrm{g} / \mathrm{m}^{3}$ and a predictive distribution $95 \%$ upper quantile of $33 \mu \mathrm{g} / \mathrm{m}^{3}$. Examination of video footage of the data collection laps revealed that these extreme levels of observed $\mathrm{PM}_{2.5}$ are often associated with being located behind large diesel vehicles, such as busses, within the CBD. To improve the representation of such observations in future model advancements, the location and movement of busses within the city centre could be modelled using bus timetable information, and by differentiating between different vehicle types in the traffic counts, discussed further in Section 5 . The $\mathrm{R}$ code and data required to fit the model in this paper is given in the Supplementary Material.

\section{Model Validation}

The results of a 10-fold cross validation of the model are presented in Figure 7, in which 10 sub samples of the data are created and recursively retained from the model for validation. In Figure 7, the true $\log \left(\mathrm{PM}_{2.5}+1\right)$ lies within the $90 \%$ prediction interval
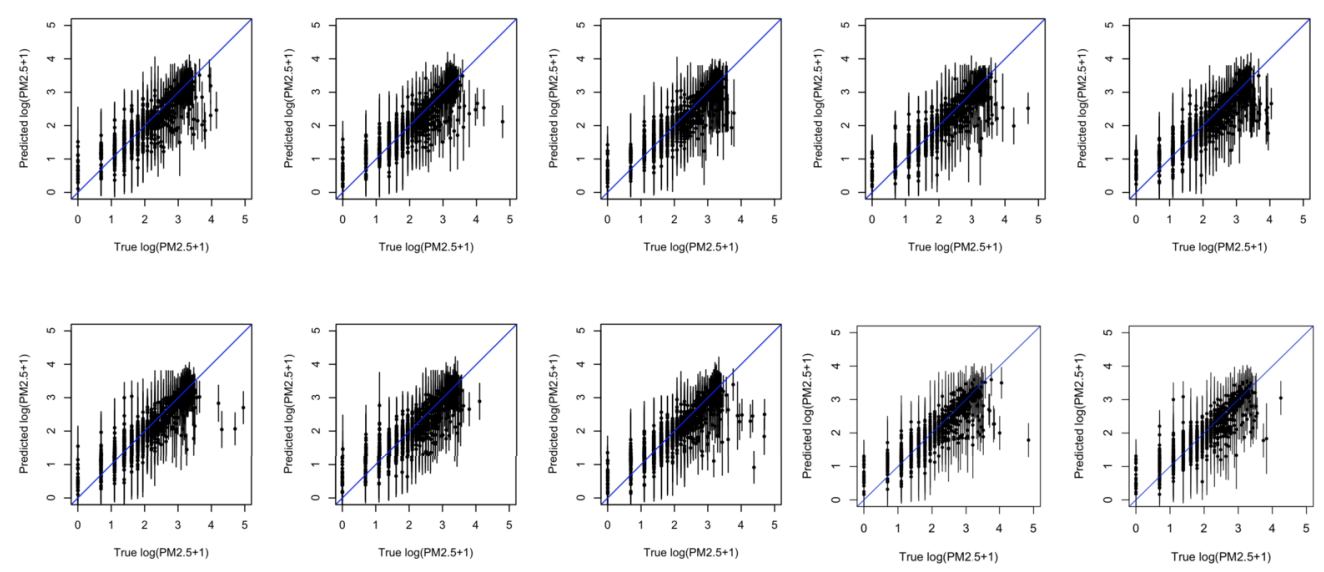

Figure 7: Scatter plots comparing the posterior predictive mean (black points) and $90 \%$ prediction interval (black line) with true $\log \left(\mathrm{PM}_{2.5}+1\right)$ for observations in each of the ten 10 -fold cross validation sub samples.

for $90.7,90.0,90.7,91.1,90.0,92.1,90.1,90.6,91.5$ and $91.2 \%$ of the observations within the validation sub sample for each of the ten plots repectively, indicating an adequate level of predictability. As identified in Figure 6, again Figure 7 shows that the model is less successful at representing higher values of $\log \left(\mathrm{PM}_{2.5}+1\right)>3.5$.

\subsection{Relating $\mathrm{PM}_{2.5}$ Exposure to Adverse Health Effects}

Communicating the risks associated with exposure to $\mathrm{PM}_{2.5}$ to the public is made most effective by relating exposure to adverse health effects. For this reason many of the existing air quality mobile phone applications and websites provide health warnings 
associated with the given air pollution measurement or forecast. These health warnings are typically based on air quality indices, calculated as the ratio of the pollution concentration and a government specified objective value. The index falls into one of five categories: Very good (0-33), Good (34-66), Fair (67-99), Poor (100-149) and Very poor $(\geq 150)$, each with associated health warnings based on numerous health studies and relevant research (Queensland Government, Accessed: 14/12/2018). For $\mathrm{PM}_{2.5}$ this government standard is based on a 24 hour moving average of the concentration recorded at that location. This approach is therefore useful when providing health warnings to an individual located in roughly the same place for 24 hours, but not as relevant when communicating the health impacts associated with instantaneous or short term changes in exposure to $\mathrm{PM}_{2.5}$, for example during a short cycle route moving from $\mathrm{A}$ to $\mathrm{B}$.

A recent study by the U.S. Environmental Protection Agency (Mannshardt et al., 2017) aimed to translate the air quality index thresholds, and the associated health warnings for the 24 hour $\mathrm{PM}_{2.5}$ standard guidelines, to those based on instantaneous air quality information. This was achieved through quantile matching of the empirical distribution of instantaneous air quality and the contemporaneous 24 hour moving average air quality. This approach, however, cannot be utilised in our study as it requires large quantities (e.g. multiple days) of continuous air quality data recorded while cycling the data collection route to adequately represent this relationship, data that was not feasible to collect here. In addition, we argue that discretising $\mathrm{PM}_{2.5}$ exposure, available on a continuous scale, using air quality index thresholds loses a great deal of detailed exposure information when describing a specific cycle route.

Alternative approaches, that do not discretise $\mathrm{PM}_{2.5}$ exposure, involve calculating the change in the relative risk $(\mathrm{RR})$ of dying from a respiratory disease as a result of a given exposure to a pollutant. In medical research, the $\mathrm{RR}$ is calculated as the probability of mortality in the exposed group divided by the probability of mortality in the unexposed group, based on large cohorts of participants, for different levels of exposure. For example, based on numerous key studies, Pope et al. (2011) used a nonlinear power function, fit to estimates of the relative risk of lung cancer mortality, cardiovascular and cardiopulmonary mortality at differing life-time daily exposures to $\mathrm{PM}_{2.5}$, to obtain functions for calculating these relative risks from daily $\mathrm{PM}_{2.5}$ dosage. The resulting equations were defined as: $\mathrm{RR}$ (lung cancer mortality $)=1+0.3195 \times\left(\text { daily } \mathrm{PM}_{2.5} \text { dose }\right)^{0.7433}$, and $\mathrm{RR}($ cardiovascular disease mortality $)=1+0.2685 \times\left(\text { daily } \mathrm{PM}_{2.5} \text { dose }\right)^{0.2730}$.

In both equations the $\mathrm{PM}_{2.5}$ dose is measured in $\mathrm{mg} / \mathrm{m}^{3}=1000 \mu \mathrm{g} / \mathrm{m}^{3}$, hence, cycling a short route through Brisbane city centre from A to B, resulting in typically no more than an increase of $0.014 \mu \mathrm{g} / \mathrm{m}^{3}=0.000014 \mathrm{mg} / \mathrm{m}^{3}$ when averaged over 24 hours, gives a $0.0079 \%$ and $1.27 \%$ increase in risk of mortality from lung cancer and cardiovascular disease respectively. These equations, however, represent the RR based on this change in daily $\mathrm{PM}_{2.5}$ dosage being experienced every day throughout an individual's life. Hence, this approach is not relevant for communicating the health risk specifically associated with taking a given cycle route as a one off. In many cases, however, a cyclist will take the same route a number of times in a week, something that could be learnt through repeated use of the cycle route decision tool. In these cases, therefore, such a $\mathrm{RR}$ health measure may be relevant in quantifying the health impact of this accumulated additional exposure to $\mathrm{PM}_{2.5}$ over a life-time, as even a $1 \%$ increase in mortality 


\section{A Bayesian Decision Framework for Personalised Cycle Route Selection}

risk will have an important impact on an individual's health. Further, the number of people affected within a population makes this a large scale and important issue.

An alternative approach for communicating the health risk from $\mathrm{PM}_{2.5}$ exposure is to associate $\mathrm{PM}_{2.5}$ dosage with the equivalent number of cigarettes smoked. Pope et al. (2011) identified that, based on the quantity of tar (a source of $\mathrm{PM}_{2.5}$ ) within a cigarette as reported by National Cancer Institute (2001), a single cigarette contains an average inhaled dose of $12 \mathrm{mg}$ of $\mathrm{PM}_{2.5}$. Therefore, using the example above, this cycle route is equivalent to $0.0168 \%$ of a cigarette. Therefore, this is a very small number that may not be useful for communicating the health risks associated with a short cycle route. Hence, none of the aforementioned approaches are directly relevant for communicating the health risk associated with a taking a short cycle route from A to B, as required within this study. Indeed, these approaches are all related to long term effects, and little data is available on short term effects resulting from short exposures to very high concentrations. This highlights the importance of further research to understand the health impact of instantaneous and short term exposures to $\mathrm{PM}_{2.5}$.

For this exercise, therefore, an alternative, simple approach is taken. Firstly, the route selection tool informs the individual of the risks associated with exposure to $\mathrm{PM}_{2.5}$ using a series of facts and figures relevant for their location. Examples of such health impact facts and figures, relevant for Brisbane and consistent with the information provided by Queensland Government (Queensland Government, Accessed: 14/12/2018) are shown in the top right corner of Figure 9. Secondly, the tool provides a measure of the average difference between the background 24 hour average $\mathrm{PM}_{2.5}$ concentration in their location, and their exposure to $\mathrm{PM}_{2.5}$ along a given journey. That is for given journey $m$, the health impact measure, $H_{m}$, is:

$$
H_{m}=\int_{t}\left(E_{m}(t)-G\right) d t,
$$

where $E_{m}(t)$ is $\mathrm{PM}_{2.5}$ exposure at time $t$ for journey $m$, and $G$ is the background 24 hour average $\mathrm{PM}_{2.5}$ level calculated from the nearest stationary air quality monitoring station. Here, this integral is approximated by summing over predicted $\mathrm{PM}_{2.5}$ from the spatio-temporal INLA barrier model at 5 second intervals, inline with the temporal resolution of the observation data used to train the model.

This measure quantifies how taking a certain route effects an individual's personal exposure to $\mathrm{PM}_{2.5}$ within that journey period. This change in $\mathrm{PM}_{2.5}$ exposure can then be related to health impacts using the facts and figures provided, and can be used to consistently compare the health impacts of travelling via different routes and at different times of the day within the Bayesian decision framework. It should be noted that this measure has the potential to be negative, indicating improved health, since cycling in a park or along a river is likely to relate to $\mathrm{PM}_{2.5}$ exposures lower than the background average level.

Figure 8 presents an example of predicted health impact of two different journeys, both based on taking route 1 in Figure 1 on Friday $25^{\text {th }}$ May 2018, but at different times: one in the afternoon time slot (15:30-17:00), journey $A$ (left column), and one 
(a)

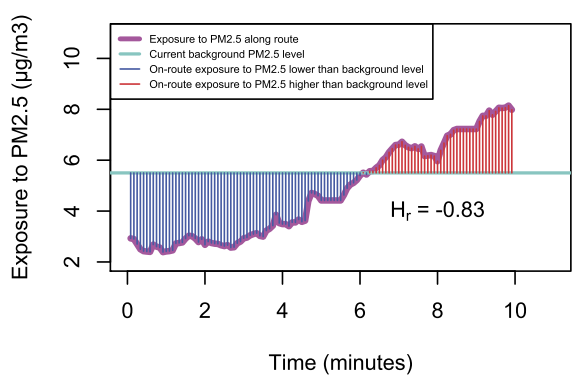

(c)

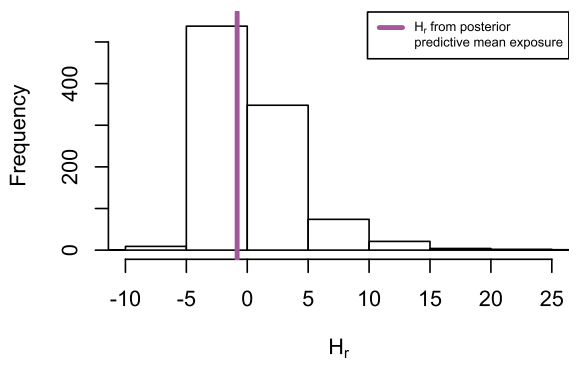

(b)

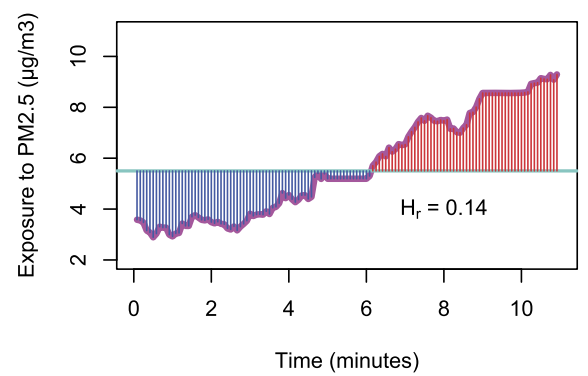

(d)

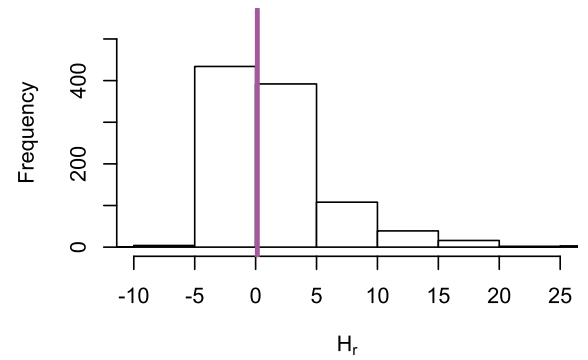

Figure 8: Graphical representation of the health impact of travelling through Brisbane city centre on Friday $25^{\text {th }}$ May 2018 via route 1 in Figure 1, during two different time slots: (a) and (c) Journey $A$ - the afternoon time slot (15:30-17:00), and (b) and (d) Journey $B$ - and the evening time slot (17:00-18:30). Figures (a) and (b) show how the posterior predicted mean exposure to $\mathrm{PM}_{2.5}$ varies at 5 second intervals along journeys $A$ and $B$ respectively. Figures (c) and (d) show histograms of health impact calculated over the full posterior predictive distribution of $\mathrm{PM}_{2.5}$ for journeys $A$ and $B$ respectively. The corresponding health impact associated with the posterior mean exposure (top row) is represented by the pink line in histograms (bottom row).

in the evening time slot, journey $B$ (right column). During journey $A$ traffic counts are lower and weather conditions favour better air quality, leading to lower predicted $\mathrm{PM}_{2.5}$ along this journey compared to journey $B$. As a result, the mean exposure to $\mathrm{PM}_{2.5}$ along journey $A$ compared to background level $(G=5.5)$ leads to a negative health impact value, $H_{A}=-0.83$, indicating a reduced health impact compared to inhaling the background level of $\mathrm{PM}_{2.5}$, while for journey $B, H_{B}=0.14$, indicating an increased health impact compared to inhaling the background level of $\mathrm{PM}_{2.5}$. Within the Bayesian decision framework, the full posterior predictive distribution for $\mathrm{PM}_{2.5}$ is used within (1) to calculate a distribution of health impacts for each journey $\left(r_{1}(d, \theta)\right)$. This ensures the optimal decision is made based on a comprehensive quantification of uncertainty. Figure 8 (c) and (d) show these health impact posterior predictive distributions for journeys $A$ and $B$ respectively. These posterior distributions are wide, both over lapping zero. However, exploration of the QQ-plots of these posterior distributions and for other pairs of journeys identifies a linear relationship, indicating that routes with larger health 


\section{A Bayesian Decision Framework for Personalised Cycle Route Selection}

impacts based on the posterior predictive mean $\mathrm{PM}_{2.5}$ will also have the highest health impact when integrated over the full distribution. Approaches for reducing this posterior spread are discussed further in Section 5.

\section{Eliciting the Utility Function}

Referring back to (1), thus far in our Bayesian decision framework for cycle route selection we have a method for estimating the state of nature $\theta$, i.e. $\mathrm{PM}_{2.5}$ exposure along the route, from available observations of $\mathrm{PM}_{2.5}, y$; a representation of all possible decisions, $d$, in the form of cycle routes from origin to destination; and an approach for representing the decision-relevant outcomes, $r_{1}(d, \theta)$, the health impact of exposure to $\mathrm{PM}_{2.5}$ along a given route, and $r_{2}(d, \theta)$ and $r_{3}(d, \theta)$, representations of the journey time and journey enjoyment. The remaining elements required from the framework are the multiattribute utility function, $U$, and the criterion weights, $k$, which must be elicited from the decision maker to represent their relevant personal preferences about the decision-relevant attributes.

In this study the aim is to develop a cycle route selection tool that could be used by any member of the general public within a smart phone application or web page. For practicality, the utility function must therefore be elicited based on only a limited series of questions, using clear, non-technical language, and visualisations to ensure understanding.

Figure 9 presents a screen shot of an RStudio Shiny web application (RStudio, Inc, Accessed: 18-12-2018), created to demonstrate how this might be achieved (associated $\mathrm{R}$ code is available in the Supplementary Material). Going from left to right, the user (decision maker) initially enters the cycle journey details: where they are travelling from and to, and how flexible they are in their time of travel. Following this the personal journey preferences are elicited.

In Bayesian Decision Theory, most commonly the utility function for a given attribute $i$, is elicited from the decision maker via a series of questions associated with a set of 'gambles' (Smith, 2010). Initially the best and worst case scenarios of the relevant attribute are defined, denoted $r^{*}$ and $r^{0}$ respectively. These outcomes are assigned utilities such that $U\left(r^{0}\right)=0$ and $U\left(r^{*}\right)=1$. The utilities associated with intermediate outcomes, $r^{0}<r<r^{*}$, are then elicited by proposing a hypothetical gamble between either the certain outcome $r$ or uncertain outcomes $\left(r^{0}, r^{*}\right)$. The decision maker is asked to specify the minimum probability $\alpha(r)$ of achieving the best case, $r^{*}$, as apposed to

the worst case, $r^{0}$, for which they would take this gamble. This minimum probability is equivalent to the utility of that intermediate outcome. (Note the term "gamble" here refers to a decision with a known set of outcomes with given probabilities, each associated with a known reward; as is common in the Bayesian decision theory literature e.g. DeGroot 1970; Smith 2010).

Therefore, firstly the Shiny decision tool presents the decision-relevant attributes and their best and worst case scenarios, specified within the 'Journey Outcomes' table (as shown in Figure 9). 


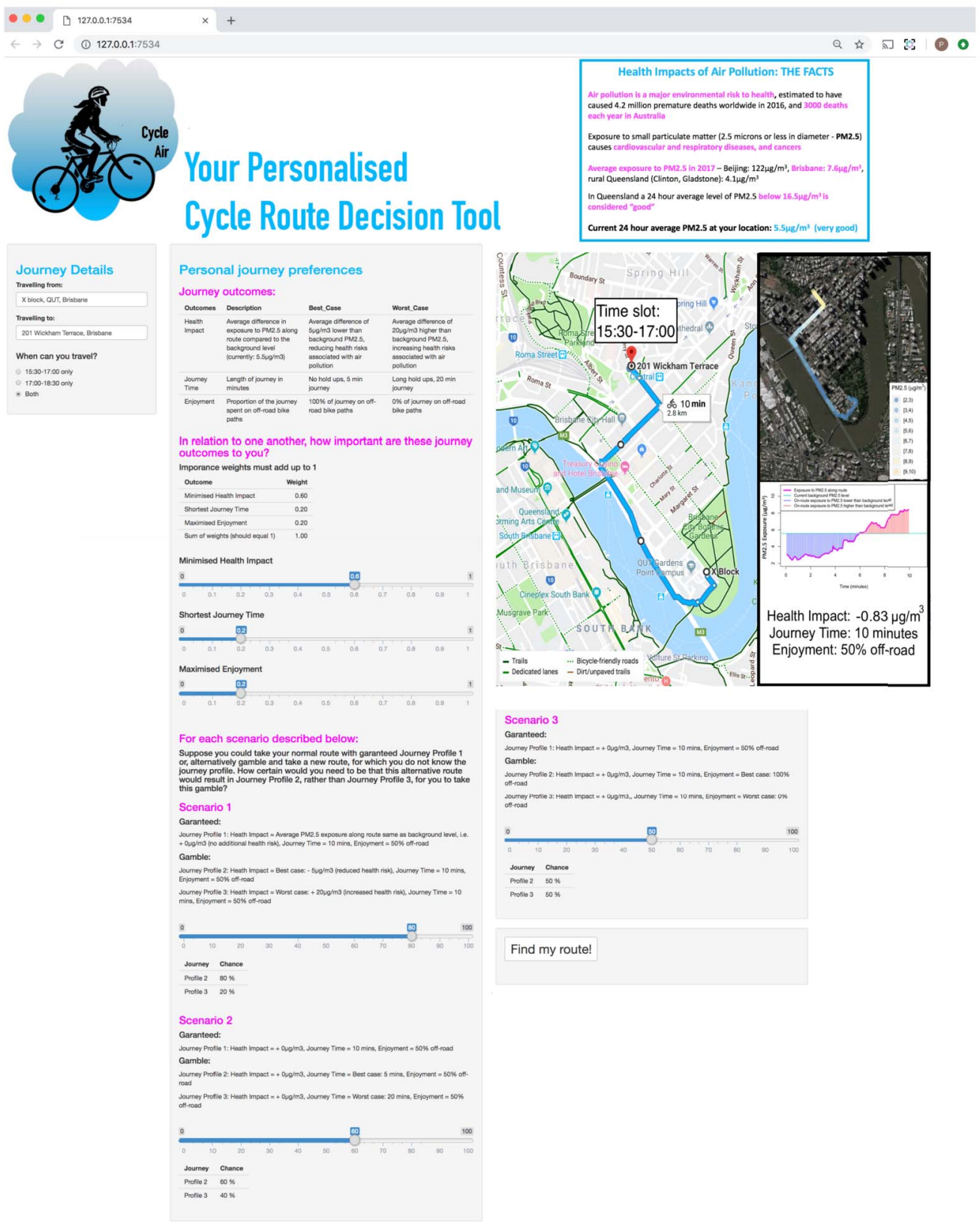

Figure 9: A screen shot of the RStudio Shiny web application developed to demonstrate how the Bayesian decision framework developed within this study could be used for personalised cycle route selection. The associated $\mathrm{R}$ code is available in the Supplementary Material. 


\section{A Bayesian Decision Framework for Personalised Cycle Route Selection}

Subsequently, the criterion weights $\left(k_{1}, k_{2}, k_{3}\right)$ are elicited from the user. This question requires the user to specify a weight for each of the decision-relevant attributes, representing how important each of these journey attributes is to them in relation to one another. The shiny allows for these weights to be allocated via sliders, coded to automatically sum to one, ensuring that the user provides a valid set of criterion weights (i.e. $\sum_{i} k_{i}=1$ ). In addition, a summary table is presented above the sliders, providing the user with a clear visualisation of their nominate weights. In the example shown in Figure $9,\left(k_{1}, k_{2}, k_{3}\right)=(0.6,0.2,0.2)$.

Next, the utility function for each attribute is elicited under the assumption of preferential independence. This allows for the utility function of each attribute to be elicited one-by-one, holding the value of all other attributes at some 'typical' value. To reduce the length and complexity of the elicitation process, for each decision-relevant attribute this involves only eliciting one point on the utility curve, in between the best and worst case scenario, via a single gamble between certain and uncertain journey outcomes (as described above). To communicate the elicitation exercise without using complex statistical terminology the three gambles are introduced as scenarios in which different outcomes are described as 'Journey Profiles' and the term 'probability' is reworded as 'certainty'. The user is asked the following:

'Suppose you could take your normal route with guaranteed Journey Profile 1 or, alternatively gamble and take a new route, for which you do not know the journey profile. How certain would you need to be that this alternative route would result in Journey Profile 2, rather than Journey Profile 3, for you to take this gamble?'. For the $i^{\text {th }}$ decision-relevant attribute, guaranteed Journey Profile 1 characterises an outcome in which that attribute takes a single value we wish to elicit the utility for, $r_{i}$, while uncertain Journey Profiles 2 and 3 characterise the best and worst case scenarios for that attribute, $\left(r_{i}^{*}, r_{i}^{0}\right)$, respectively. In all profiles the other two attributes are held at a medium outcome. The required certainty/probability of Journey Profile 2 is specified by the user via a slider ranging from $0-100 \%$. Again, a summary table is presented with each scenario to clearly communicate to the user the response associated with their slider setting.

Since the best and worse case scenarios are assigned utility 1 and 0 respectively, following this elicitation exercise we have three points on the utility curve for each attribute. For attribute 1 (health impact) in Figure $9, r_{1}=(-5,0,20)$ and $U\left(r_{1}\right)=$ $(1,0.8,0)$, where 0.8 is taken from associated slider (divided by 100). Similarly, for attribute 2 (journey time), $r_{2}=(5,10,20)$ and $U\left(r_{2}\right)=(1,0.6,0)$, and attribute 3 (Enjoyment, i.e. proportion of the journey spent off-road), $r_{3}=(1,0.5,0)$ and $U\left(r_{3}\right)=$ $(1,0.5,0)$. For each attribute, the full utility curve is then calculated by fitting a piecewise logistic function, $U\left(r_{1 j}\right)=a \log \left(r_{1 j}\right)+b$, to each pair of points on the curve, $j=(1,2)$ and $j=(2,3)$. An alternative, more sophisticated approach such as Gaussian processes could be used to fit the utility function in future revision of this decision framework, however this simplistic approach was considered to be adequate for this demonstration. Figure 10 (a)-(c) presents univariate utility functions for the three jour- 
ney decision-relevant attributes, each represented as a piecewise logistic function fit to the elicited values in Figure 9.

(a)

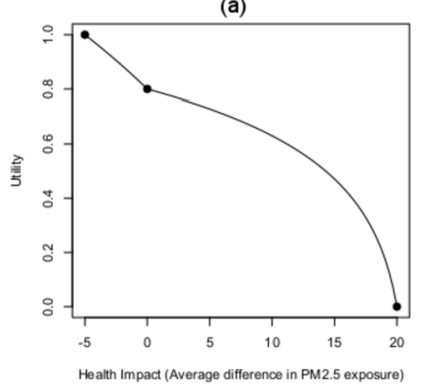

(d)

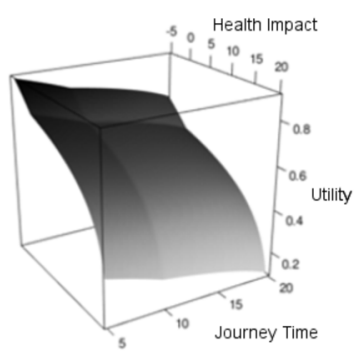

(b)

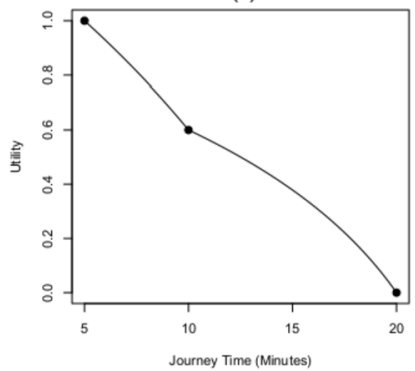

(e)

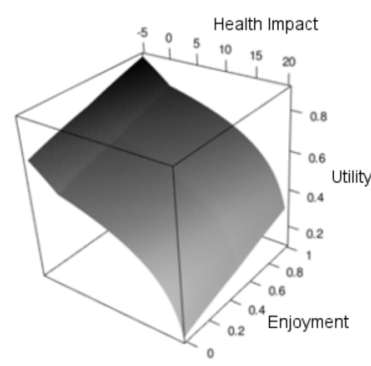

(c)

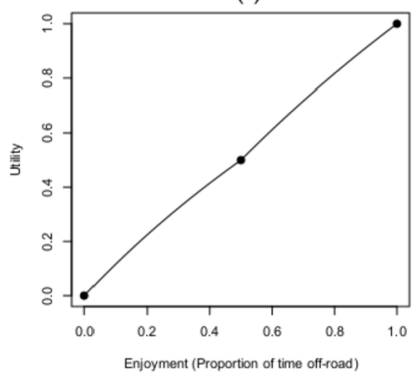

(f)

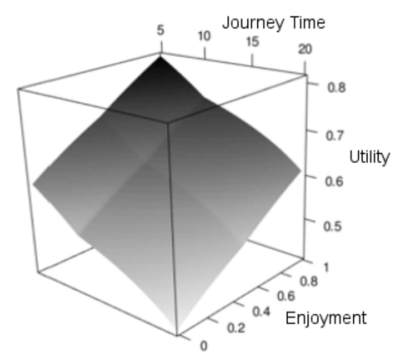

Figure 10: Top row: Univariate utility functions for each of the cycle journey decisionrelevant attributes (a) health impact, (b) journey time, (c) enjoyment. The black points represent the three points elicited from the R shiny web application (Figure 9) and the adjoining curves represent the piecewise logistic functions fit to represent the utility function for each attribute. Bottom row: A 3-dimensional representation of the multidimensional utility surface, where (d) enjoyment, (e) journey time, (f) health impact is held at it's mean value to demonstrate how the surface varies with the remaining two attributes.

Finally, following the assumption of mutual independence, the multiattribute utility function is calculated as the criterion weighted sum of the three univariate utility functions, as in (1). Figure 10 (d)-(f) presents a 3-dimensional graphical representation of the multidimensional utility surface elicited in Figure 9. This surface characterises how the utility of a decision varies with all three attributes, given this specific user's personal preferences.

This assumption of mutual independence, which requires the utility function of each attribute to be independent of all other attributes, can be modified. For example, the importance of avoiding poor air quality may vary if an individual is considering journeys with different journey times. Possible approaches for accommodating mutual dependence are discussed further in Section 5. 


\section{A Bayesian Decision Framework for Personalised Cycle Route Selection}

\section{Case Study}

We now present a demonstration of the full Bayesian personalised air quality decision framework. This demonstration uses the case study routes shown in Figure 1, each of which could be cycled in the afternoon time slot (15:30-17:00) or the evening time slot (17:00-18:30), resulting in four possible journeys, with decision-relevant attributes:

1. Journey A: Route 1, 15:30-17:00

- Mean Health Impact: $-0.56 \mu \mathrm{g} / \mathrm{m}^{3}$

- Journey Time: 10 minutes

- Enjoyment: 0.5 (proportion of journey off-road)

2. Journey B: Route 1, 17:00-18:30

- Mean Health Impact: $0.44 \mu \mathrm{g} / \mathrm{m}^{3}$

- Journey Time: 11 minutes

- Enjoyment: 0.45

3. Journey C: Route 2, 15:30-17:00

- Mean Health Impact: $-0.29 \mu \mathrm{g} / \mathrm{m}^{3}$

- Journey Time: 8.5 minutes

- Enjoyment: 0.24

4. Journey D: Route 2, 17:00-18:30

- Mean Health Impact: $1.28 \mu \mathrm{g} / \mathrm{m}^{3}$

- Journey Time: 10 minutes

- Enjoyment: 0.2

These values are based on the $\mathrm{PM}_{2.5}$ concentrations predicted for unobserved day Friday $25^{\text {th }}$ May 2018, and use the Google Maps route planner to determine journey time and progression. The slight differences in journey time and enjoyment in the two time slots is due to an expected extra waiting time at traffic lights during the later time slot as a result of greater volumes of commuter traffic after 17:00.

From these four potential journeys, each with their differing decision-relevant attributes, the user specified utility surface will dictate which is identified as the optimal journey. For example, for the utility surface elicited in Figure 9, shown in Figure 10, in which the user is most concerned with minimising the health impact of the journey followed by minimising the journey time,

$$
\begin{aligned}
\left(k_{1}, k_{2}, k_{3}\right) & =(0.6,0.2,0.2), \\
U_{1}(-5,0,20) & =(1,0.8,0), \\
U_{2}(5,10,20) & =(1,0.6,0), \\
U_{3}(0,0.5,1) & =(0,0.5,1) .
\end{aligned}
$$


Then for each journey (A-D) specified above, the utility is calculated as the sum of individual journey attribute utilities (as in (1)):

$$
\begin{aligned}
\text { Journey A: } U\left(r_{A}\right) & =0.6 U_{1}(-0.56)+0.2 U_{2}(10)+0.2 U_{3}(0.5) \\
& =0.716 \\
\text { Journey B: } U\left(r_{B}\right) & =0.6 U_{1}(0.44)+0.2 U_{2}(11)+0.2 U_{3}(0.45) \\
& =0.682 \\
\text { Journey C: } U\left(r_{C}\right) & =0.6 U_{1}(-0.29)+0.2 U_{2}(8.5)+0.2 U_{3}(0.24) \\
& =0.686 \\
\text { Journey D: } U\left(r_{D}\right) & =0.6 U_{1}(1.28)+0.2 U_{2}(10)+0.2 U_{3}(0.2) \\
& =0.635
\end{aligned}
$$

To ensure the optimal journey is selected based on the full spread of health impact journey outcomes, calculated from the full posterior of the INLA model for $\mathrm{PM}_{2.5}$, the expected utility of each journey $(\mathrm{A}-\mathrm{D})$ is calculated instead. Rather than using the mean health impact for each journey as in the above equations, the utility for the health impact attribute $\left(U_{1}\right)$ is integrated over all posterior samples, as in (1). In this example the journey time and enjoyment attributes are treated as deterministic, however future developments of this framework could incorporate uncertainty in these journey attributes. The resulting expected utilities for each of the journeys (A, B, C, D) are calculated as $(0.712,0.681,0.684,0.628)$ respectively, indicating that this user should cycle Journey A (Route 1, 15:30-17:00), since this journey has maximised the expected utility. This makes intuitive sense, since this route is predominantly cycled along the river, where $\mathrm{PM}_{2.5}$ exposure is generally lower, in the earlier time slot when $\mathrm{PM}_{2.5}$ levels are lower and the journey can be completed more quickly.

In an alternative scenario, the user may be most concerned with arriving at their destination as quickly as possible and have less interest in minimising health impact. In this case the user may nominate:

$$
\begin{aligned}
\left(k_{1}, k_{2}, k_{3}\right) & =(0.3,0.6,0.1), \\
U_{1}(-5,0,20) & =(1,0.65,0), \\
U_{2}(5,10,20) & =(1,0.8,0), \\
U_{3}(0,0.5,1) & =(0,0.5,1),
\end{aligned}
$$

resulting in expected utility for each journey $(\mathrm{A}, \mathrm{B}, \mathrm{C}, \mathrm{D})$ equal to $(0.660,0.623,0.708$, 0.614 ) respectively. This now indicates that the user should take Journey C (Route 2, 15:30-17:00) as this journey has maximised the expected utility. Again, this makes sense because this is journey is the fastest of the four, but is travelled in the earlier time slot so the exposure to $\mathrm{PM}_{2.5}$ is still moderate.

Further variations in the elicited information result in alternative route selections. For example, an individual who must leave work at 17:00 is restricted to travelling in the evening time slot, resulting in the identification of optimal routes B or D depending on additional journey preferences. 


\section{A Bayesian Decision Framework for Personalised Cycle Route Selection}

This case study has focused on one user and four potential journeys only. In order to make this methodology operationally feasible it must be scaled up to accommodate a larger number of cyclists, more journey routes and times, and additional decision relevant journey attributes (e.g. safety), while ensuring realistic-time updating of the model. The most demanding computation is the prediction of the $\mathrm{PM}_{2.5}$ spatial field from the INLA model (currently taking approximately 15 minutes on a laptop). This spatial field would be the same for all users of the route decision tool in the same city, and could therefore be continually updated, based on air quality and weather forecast information, and facilitate all user's route calculations. This would therefore require a forecast feed to be set up as an input to the INLA model, which could be run every few minutes, perhaps on a high speed server or super-computer to reduce the computation time. For each cyclist, the consideration of more routes and journey times would then be relatively computationally cheap, simply requiring the quick calculation of the journey time, proportion of the journey off-road, and the exposure to $\mathrm{PM}_{2.5}$ during the journey. Operationally, these journey characteristics could be calculated more efficiently by linking the app to a web mapping service (e.g. Google Maps).

\section{Conclusion}

This study demonstrates an approach using Bayesian methods to support personalised decision making for air quality. This is achieved by developing a novel and effective methodology which combines hierarchical spatio-temporal modelling of city air quality, incorporating the effect of covariates and representing buildings as barriers, and a multiattribute Bayesian decision framework, to provide Bayes-optimal decision guidance. This proof-of-concept application demonstrates the extremely important role Bayesian approaches could have in the future of personalised decision making, since Bayesian uncertainty quantification leads to meaningful decision making. As such, the presented methodology has the potential to be deployed in any town or city, and for any mode of transport. A council could engage citizens to complete many routes, all of which could be integrated within such a framework and used to produce online inference that can be accessed by users via a smart phone application. This all feeds into the future vision of a smart city, such as that discussed by Barr et al. (2019), where citizens make informed and engaged choices about their mobility. The output of such a framework could also be used to inform city planning, for example the planning of bus routes to minimise impact on public health.

The current methodology has the potential to be further developed, beyond this proof-of-concept demonstration, in a number of ways. The hierarchical spatio-temporal model (2) includes error terms at each level of the hierarchy. These are used to account for the variance within the data not captured by the covariates, and subsequently result in model predictions with large posterior variances. When $\mathrm{PM}_{2.5}$ predictions are added up along a route, these combined variances result in a large spread in the predicted health impact for a given journey (as seen in Section 2.3). To reduce this posterior spread additional, more relevant covariates could be incorporated into the model. For example, incorporating traffic counts from all intersections in the city centre. In addition, a linear relationship is assumed between each covariate and response variable. Future model 
configurations should allow for non-linear relationships, for example using penalised splines.

Moreover, here, due to time constraints of the project, the model for $\mathrm{PM}_{2.5}$ is based on just 48 laps of the data collection route. Additional laps would likely reduce the posterior spread of parameter estimates. Furthermore, additional data laps could be used to increase the temporal resolution of the model (e.g. 30 minute rather than 1.5 hour time slots). The current approach of averaging covariates over a 1.5 hour time slot, in which meteorological conditions can change drastically, results in a loss of detail which, if retained, could improve model predictability. In addition, the data used in this study is representative of the month of May only. Further development would require data to be collected in all months of the year, potentially requiring the inclusion of alternative meteorological covariates, with differing relationships for each month.

The current model does not successfully represents very extreme levels of $\mathrm{PM}_{2.5}$ $\left(>46 \mu \mathrm{g} / \mathrm{m}^{3}\right)$. These extreme values were identified as times when the data collection cyclists were located behind large diesel vehicles, most often buses, and are important to capture in the model to ensure accurate journey comparison within the decision framework. Potential approaches for better representing these extremes could involve differentiating between traffic counts from large and small vehicles or incorporating information from bus time tables as an additional covariate for 'distance from bus'.

Within the decision tool the health impact of exposure to $\mathrm{PM}_{2.5}$ is quantified as a measure of the overall difference between exposure along the journey compared to the background $\mathrm{PM}_{2.5}$ level, related to a series of health facts and figures. The effectiveness of the decision tool would be improved by incorporating further insights from essential future research into how instantaneous exposures to varying $\mathrm{PM}_{2.5}$ over short journeys effects health in a more direct, tangible way. In addition, the improved measure of health impact should differentiate between individuals with pre-existing cardiovascular conditions and are therefore more vulnerable to exposure to $\mathrm{PM}_{2.5}$, as well as differences in gender and age groups. Further, following model development to better represent extreme levels of $\mathrm{PM}_{2.5}$, information such as the probability of exposure to these extreme levels, known to cause instantaneous health impacts, could be included.

Furthermore, The assumption of mutual independence between journey attributes within the Bayesian decision framework may be an over simplification here. For example, an individual may be less concerned about journey time if that journey has a high value for enjoyment. To capture this dependence between journey attributes, whilst also keeping the elicitation process manageable within a mobile phone application, the tool could initially ask a basic set of elicitation questions, as described in this study, and then build up a more complex utility surface by asking additional questions between varying journey attributes each time the users interacts with the app, and based on the users actual decision over a number of days. In addition, collaboration with social scientists could improve communication within the tool, facilitating theoretically sound elicitation using the most easily understandable language possible. 


\section{A Bayesian Decision Framework for Personalised Cycle Route Selection}

\section{Supplementary Material}

Supplementary Material (DOI: 10.1214/19-BA1193SUPP; .zip).

\section{References}

Australian Institute of Health and Welfare (2016). "Australian Burden of Disease Study: impact and causes of illness and death in Australia 2011." Technical report, Australian Government. 61

Bakka, H., Rue, H., Fuglstad, G., Riebler, A., Bolin, D., Illian, J., Krainski, E., Simpson, D., and Lindgren, F. (2018). "Spatial modeling with R-INLA: A review." Computational Statistics, 10(6, e1443). MR3873676. doi: https://doi.org/10.1002/wics. 1443. 63,71

Bakka, H., Vanhatalo, J., Illian, J. B., Simpson, D., and Rue, H. (2019). "Non-stationary Gaussian models with physical barriers." Spatial Statistics, 29: 268-288. MR3903698. doi: https://doi.org/10.1016/j.spasta.2019.01.002. 71

Barr, S. W., Lampkin, S. R., Williamson, D. B., and Dawkins, L. C. (2019). "Smart cities and behavioural change: (re)empowering mobilities." Transactions of the Institute of British Geographers. In review. 86

BreezoMeter (Accessed: 27-11-2018). "BreezoMeter." https://breezometer.com/. 62

Brisbane Times (Accessed: 14-01-2019). "Brisbane cycling data." https://www. brisbanetimes.com.au/national/queensland/brisbane-cycling-data-reveals -when-and-where-people-hop-on-their-bikes-20170626-gwywi2.html. 66

Cocchi, D., Greco, F., and Trivisano, C. (2007). "Hierarchical space-time modelling of PM10 pollution." Atmospheric Environment, 41(3): 532-542. 63

Dawkins, L. C., Williamson, D. B., Mengersen, K. L., Morawska, L., Jayaratne, R., Shaddick, G. (2020). "Where is the clean air? A Bayesian decision framework for personalised cyclist route selection using R-INLA - Supplementary Material." Bayesian Analysis. doi: https://doi.org/10.1214/19-BA1193SUPP. 73

DeGroot, M. H. (1970). Optimal Statistical Decisions. McGraw-Hill, New York. MR0356303. 80

Del Sarto, S., Ranalli, M. G., Cappelletti, D., Moroni, B., Crocchianti, S., and Castellini, S. (2016). "Modelling spatio-temporal air pollution data from a mobile monitoring station." Journal of Statistical Computation and Simulation, 86(13): 2546-2559. MR3511012. doi: https://doi.org/10.1080/00949655.2016.1167895. 63,69,70, 71

Economou, T., Stephenson, D. B., Rougier, J. C., Neal, R. A., and Mylne, K. R. (2016). "On the use of Bayesian decision theory for issuing natural hazard warnings." Proceedings of the Royal Society A, 472(20160295). MR3592330. doi: https://doi.org/ 10.1098/rspa.2016.0295. 62 
Elen, B., Peters, J., Van Poppel, M., Bleux, N., Theunis, J., Reggente, M., and Standaert, A. (2013). "The Aeroflex: a bicycle for mobile air quality measurements." Sensors, 13: 221-240. 63

Faraway, J. (Accessed: 13/12/2018). "INLA analysis of a multilevel model." http:// www.maths.bath.ac.uk/ jjf23/inla/multilevel.html. 73

Gelman, A., Hwang, J., and Vehtari, A. (2014). "Understanding predictive information criteria for Bayesian models." Statistics and Computing, 24(6): 997-1016. MR3253850. doi: https://doi.org/10.1007/s11222-013-9416-2. 71

Gelman, A., Jakulin, A., Grazia Pittau, M., and Su, Y. (2008). "A weakly informative default prior distribution for logistic and other regression models." The Annals of Applied Statistics, 2(4): 1360-1383. MR2655663. doi: https://doi.org/10.1214/ 08-AOAS191. 72

Hoek, G., Beelen, R., de Hoogh, K., Vienneau, D., Gulliver, J., Fischer, P., and Briggs, D. (2008). "A review of land-use regression models to assess spatial variation of outdoor air pollution." Atmospheric Environment, 42(7561-7578). 69

Hoek, G., Krishnan, R. M., Beelen, R., Peters, A., Ostro, B., Brunekreef, B., and Kaufman, J. D. (2013). "Long-term air pollution exposure and cardio-respiratory mortality: a review." Environmental Health, 12(article 43). 61

ILAQH (Accessed: 28-11-2018). "The Gold Coast, its air - and KOALAs." https:// research.qut.edu.au/ilaqh/projects/thegoldcoastanditsair/. 63, 65

Kumar Mehta, S., Venkat Ratnam, M., Sunilkumar, S. V., Narayana Rao, D., and Krishna Murthy, B. V. (2017). "Diurnal variability of the atmospheric boundary layer height over a tropical station in the Indian monsoon region." Atmospheric Chemistry and Physics, 17: 531-549. 72

Li, L., Wu, J., Ghosh, J., and Ritz, B. (2013). "Estimating spatiotemporal variability of ambient air pollutant concentrations with a hierarchical model." Atmospheric Environment, 71(54-63). 63

Lindgren, F. and Rue, H. (2011). "An explicit link between Gaussian fields and Gaussian Markov random fields: the stochasticpartial differential equation approach." Journal of the Royal Statistical Society: Series B, 73(4): 423-498. MR2853727. doi: https:// doi.org/10.1111/j.1467-9868.2011.00777.x. 70, 71

Loomis, D., Grosse, Y., Lauby-Secretan, B., El Ghissassi, F., Bouvard, V., BenbrahimTallaa, L., Guha, N., Baan, R., Mattock, H., and Straif, K. (2013). "The carcinogenicity of outdoor air pollution." The Lancet Oncology, 14: 1262-1263. 61

Mannshardt, E., Benedict, K., Jenkins, S., Keating, M., Mintz, D., Stone, S., and Wayland, R. (2017). "Analysis of short-term ozone and PM2.5 measurements: Characteristics and relationships for air sensor messaging." Journal of the Air $\&$ Waste Management Association, 67(4): 462-474. 77

MappAir (Accessed: 27-11-2018). "MappAir Website." https://www.earthsense.co. $\mathrm{uk} / \mathrm{bbc}$-mappair-faq. 62 


\section{A Bayesian Decision Framework for Personalised Cycle Route Selection}

Mateo-Babiano, I., Bean, R., Corcoran, J., and Pojani, D. (2016). "How does our natural and built environment affect the use of bicycle sharing?" Transportation Research Part A, 94: 295-307. 66, 67

Morawska, L., Jayaratne, R., Mengersen, K. L., Jamriska, M., and Thomas, S. B. (2002). "Differences in airborne particle and gaseous concentrations in urban air between weekdays and weekends." Atmospheric Environment, 36(27): 4375-4383. 71

National Cancer Institute (2001). "Risks Associated with Smoking Cigarettes with Low Machine-Measured Yields of Tar and Nicotine. Smoking and Tobacco Control Monograph No. 13." Technical report, NIH Pub. No. 02-5047 Bethesda, MD:National Institutes of Health. 78

Peters, J., Theunis, J., Van Poppel, M., and Berghmans, P. (2013). "Monitoring PM10 and ultrafine particles in urban environments using mobile measurements." Aerosol Air Quality Research, 13: 509-522. 66, 67

Plume Labs (Accessed: 27-11-2018). "Plume Labs Website." https://plumelabs.com/ en/. 62

Pope, C. A. I., Burnett, R. T., Turner, M. C., Cohen, A., Krewski, D., Jerrett, M., Gapstur, S. M., and Thun, M. J. (2011). "Lung cancer and cardiovascular disease mortality associated with ambient air pollution and cigarette smoke: shape of the exposure-response relationships." Environmental Health Perspectives, 119(11): 16161621. 77,78

Queensland Government (Accessed: 14/12/2018). "Queensland Government Hourly Air Quality data." https://environment.des.qld.gov.au/air/data/search.php. $62,68,77,78$

RStudio, Inc (Accessed: 18-12-2018). Easy web applications in R. URL http://www. rstudio.com/shiny. 80

Rue, H., Martino, S., and Chopin, N. (2009). "Bayesian inference for latent Gaussian models by using integrated nested Laplace approximations." Journal of the Royal Statistical Society: Series B, 71(2): 319-392. MR2649602. doi: https://doi.org/ $10.1111 / j .1467-9868.2008 .00700$.x. 63

Sahu, S. and Bakar, K. (2012). "Hierarchical Bayesian autoregressive models for large space-time data with applications to ozone concentration modelling." Applied Stochastic Models in Business and Industry, 28(5): 395-415. MR2990227. doi: https://doi . org/10.1002/asmb.1951. 63

Sava, F. and Carlsten, C. (2012). "Respiratory health effects of ambient air pollution: an update." Clinics in Chest Medicine, 33: 759-769. 61

Shaddick, G. and Zidek, J. V. (2014). "A case study in preferential sampling: Long term monitoring of air pollution in the UK." Spatial Statistics, 9: 51-65. MR3326831. doi: https://doi.org/10.1016/j.spasta.2014.03.008. 71

Smith, J. Q. (2010). Bayesian Decision Analysis: Principles and Practice. Cam- 
bridge University Press, New York. MR2828346. doi: https://doi.org/10.1017/ CB09780511779237. 62, 65, 80

Strava (Accessed: 14-01-2019). "Strava Global Heatmap." https : //www. strava.com/ heatmap\#11.95/153.01155/-27.45833/hot/all. 66

Van den Bossche, J., Peters, J., Verwaeren, J., Botteldooren, D., Theunis, J., and Baets, B. D. (2015). "Mobile monitoring for mapping spatial variation in urban air quality: Development and validation of a methodology based on an extensive dataset." Atmospheric Environment, 105: 148-161. 62, 63, 66, 67

World Health Organization (Accessed: 27-11-2018). "Air pollution." http://www. who . int/airpollution/en/. 61

Xie, X., Semanjski, I., Gautama, S., Tsiligianni, E., Deligiannis, N., Thilak Rajan, R., Pasveer, F., and Philips, W. (2017). "A review of urban air pollution monitoring and exposure assessment methods." International Journal of Geo-Information, 6(389). 63

Zhang, Y., Zhang, S., Huang, C., Huang, K., Gong, Y., and Gan, Q. (2014). "Diurnal variations of the planetary boundary layer height estimated from intensive radiosonde observations over Yichang, China." Science China Technological Sciences, 57(11): 2172-2176. 72

\section{Acknowledgments}

Gavin Shaddick's time on this project was funded by the Health Inequalities and Air Quality in Scotland (HEALTH-IEAIR) SECURE project. The data and R code used in this paper are available in the associated Supplementary Material. 